\title{
Efecto de una corta de protección sobre el establecimiento de regeneración en bosque de Nothofagus
}

\section{Effect of protection cut on the establishment of regeneration in Nothofagus forest}

\author{
Pablo Cruz ${ }^{*}$ y Javiera Urrutia ${ }^{2}$
}

1 Global Environment Facility. Corredores de Montaña, Ministerio de Medio Ambiente. Santiago, Chile.

\section{RESUMEN}

El objetivo de este estudio fue evaluar el efecto de una corta, sobre el establecimiento de regeneración en un bosque dominado por Nothofagus obliqua en la cordillera de los Andes de la Región del Maule, después de cuatro años de su aplicación. Fueron establecidos tres ensayos; dos en un bosque cuya cobertura de copa inicial fue de 147\% y $89 \%$, quedando después de cuatro años desde la corta con $93 \%$ y $60 \%$ con la extracción, uno de ellos plano (llamado denso sin pendiente o DsP) y el otro con pendiente suave y exposición SO (DcP). Un tercer ensayo correspondió a un área plana con apenas $10 \%$ de cobertura inicial y que no fue intervenido (Asp). Las mayores densidades de regeneración ocurrieron en la zona de bosque plana, con 8332 plantas por hectárea, mientras que en la zona con pendiente se estimó una densidad de 4592 plantas por hectárea. La zona abierta solamente alcanzó 1528 plantas por hectárea. En todos los casos hubo regeneración de especies de diferente tolerancia a la luz y el N. obliqua ocupó un valor relativo de importancia en torno a $50 \%$. Es destacable la presencia de especies del tipo forestal esclerófilo, de las cuales, Cryptocarya alba en particular ocupó un segundo lugar en importancia en el ensayo con pendiente, con casi una cuarta parte del total. Así, estos resultados son un acercamiento para entender como el manejo de la cobertura podría ser una potente herramienta de control de la composición del bosque futuro, a pesar que el período del estudio coincidió con una sequía inusual en el país y que podría haber afectado la densidad medida.

PALABRAS CLAVE: cobertura de copa, corta semillera, densidad, regeneración.

\section{ABSTRACT}

The objective of this project was to evaluate the effect of a cut, on the establishment of regeneration in a forest dominated by Nothofagus obliqua in the Andes Mountains of the Maule Region, after four years of its application. Three tests were established, two with forest canopy cover of $147 \%$ and $89 \%$ before the cut, being $93 \%$ and $60 \%$ after the extraction, one of them flat (called dense without slope or DsP) and the other with smooth slope and SW exposure (DcP). A third test corresponded to an area with few mature trees with $10 \%$ coverage (AsP). The highest densities of regeneration occurred in the area of flat forest, with 8347 seedlings (pl.) per hectare, while in the area with slope a density of 4838 plants per hectare has estimated. The open area only reached 1528 plants per hectare. In all cases there was regeneration of species with different tolerance to light, and the relative importance of N. obliqua reached about $50 \%$. Remarkable is the presence of species of forest type esclerophyl, of which, particularly Cryptocarya alba occupied second place in importance in the test with slope, with nearly a quarter of the total. Thus, these results are seen as an approach for managing the coverage which could be a powerful tool for controlling the composition of the future forest, even though the study period coincided with an unusual drought in the country and could have affected the density measured.

KEYWORDS: canopy cover, seedling cut, density, regeneration.

\section{INTRODUCCIÓN}

La dinámica regenerativa de Nothofagus obliqua (Mirb.) Oerst. se ve favorecida por perturbaciones naturales como deslizamientos del terreno, por sismos o caídas masivas de árboles por efecto del viento (Veblen y Ashton, 1978; Veblen, Schlegel y Escobar, 1980; Donoso y Lara, 1999), debido a que es una de las especies más intolerantes respecto a la sombra del grupo de Nothofagus de los bosques del centro sur de Chile. Evidencias de ello son las vastas superficies de renovales que aparecen en zonas en las que los bosques se han eliminado, ya sea accidentalmente o por abandono de la actividad agrícola (Donoso y Lara, 1999). Por esta razón, diversos autores recomiendan que el método de cosecha y 
regeneración para bosques dominados por $N$. obliqua sea el de cortas de protección (Vita, 1996; Grosse y Quiroz, 1998; Martínez-Velásquez, 1998; Otero y Monfil, 1999). No obstante, existen pocos antecedentes que precisen la dinámica de aparición, crecimiento y composición de la regeneración en este tipo de bosques, según se realice una reducción gradual de la cobertura superior del bosque. En efecto, la dinámica natural que ha sido descrita para la especie corresponde más bien a efectos de aperturas bruscas del dosel y de las que no existen datos precisos del plazo requerido para el establecimiento, conociéndose solamente que el resultado son bosques puros. Sola, Attis, Chauchard y Gallo, (2015), para bosques mixtos de Nothofagus en la Reserva Nacional Lanín, proponen un plazo de 20 años para el establecimiento de la regeneración luego de aplicadas las cortas de protección. Los mismos autores indican que la liberación de coberturas de hasta $60 \%$ aproximadamente retardó en cinco años la aparición de la regeneración, respecto a los bosques con mayor cobertura residual en la que la regeneración se inició casi inmediatamente. Otros autores han encontrado que bosques sin intervención podrían tener un banco permanente de plantas de Nothofagus a razón de 300 mil individuos por hectárea y que logran mantenerse hasta los tres años de edad si no son liberadas (Martínez-Pastur, Peri, Fernández y Staffieri, 1999; Weinberger y Ramírez, 1999).

La eliminación gradual de la cobertura como mecanismo esencial de una corta de protección, podría ser semejante a la dinámica de aperturas del dosel por caída de árboles viejos, como la describen varios autores (Veblen, 1985; Pollmann, 2004; González, Donoso y Zejner, 2015), y que genera un ambiente propicio para N. obliqua y otras especies menos intolerantes, resultando regeneraciones mixtas con $N$. obliqua. Por ejemplo, Pollmann (2004) indica que, en la cordillera de los Andes, las aperturas por caída de árboles individuales producen composiciones mixtas de Nothofagus alpina (Poepp. et Endl.) Oerst. y Laurelia philippiana (Looser) Schodde. Así también Weinberger y Ramírez (1999) proponen la existencia de una fuerte regeneración de semilla de $N$. alpina únicamente si existe un dosel protector. Los mismos autores señalan que el grado de cobertura determinará la participación de Persea lingue R. et P., podocarpáceas y otras especies tolerantes, junto a los Nothofagus. En contraste en el lado argentino en torno a la latitud $40^{\circ}$ sur, Dezzotti, Sbrancia,
Rodríguez-Arias, Roat y Parisi, (2003), en claros formados por corta con superficies entre $2000 \mathrm{~m}^{2}$ y $4000 \mathrm{~m}^{2}$, no detectaron regeneración de especies diferentes a las del género Nothofagus, las cuales alcanzaron hasta 90 mil plantas por hectárea luego de ocho años.

Así es posible afirmar que los antecedentes relativos al establecimiento de regeneración por efecto de aperturas del dosel son escasos en el país, especialmente aquellos referidos a los plazos para considerar una nueva generación establecida y las composiciones esperables. Este conocimiento es relevante si se considera que la legislación chilena para bosques de Nothofagus autoriza cortas de regeneración y cosecha mediante los cuatro métodos silvícolas conocidos: tala rasa; árbol semillero; cortas de protección y corta selectiva (Corporación Nacional Forestal, [Conaf] 1980), todos basados en una reducción de la cobertura del dosel, y para los cuales solamente se exige el establecimiento de 3000 plantas por hectárea de las mismas especies del tipo forestal, sin detallar su tiempo de vida ni el origen (semilla o vegetativa) de ella. En este contexto, la hipótesis central del estudio fue que la corta semillera, como primera corta del método silvícola de cortas sucesivas aplicado a bosques de $N$. obliqua en la cordillera de los Andes en la zona central de Chile, sería un método efectivo para lograr el establecimiento de regeneración principalmente de Nothofagus obliqua, dado que en bosques compuestos por otras especies intolerantes similares a $N$. obliqua en Europa ha sido usado con éxito durante largos años (Vita, 1996; Montoya y Mesón, 2004; González, 2005; Serrada, 2008). El concepto de método efectivo debe considerar determinar tanto el número adecuado de plantas establecidas, como el tiempo que requiere el proceso.

\section{OBJETIVOS}

El objetivo del estudio fue evaluar el efecto de una corta semillera bajo el método de corta de protección, sobre el establecimiento de la regeneración, después de cuatro años de la intervención, en bosques de $N$. obliqua en la cordillera de la Costa de la región del Maule, Chile.

\section{MATERIALES Y MÉTODOS}

Área de estudio. El estudio se realizó en la precordillera de los Andes de la Región del Maule (35³3' 41" latitud Sur; $71^{\circ} 10^{\prime} 41^{\prime}$ 
longitud Oeste; altitud promedio de $1300 \mathrm{~m}$ snm), en un área de 2 ha (Fig. 1). El clima de la zona es templado cálido con lluvias invernales (Csb), según la clasificación de Köppen-Geiger (Merkel, 2018). La zona registra precipitaciones totales en torno a $1168 \mathrm{~mm}$ al año y, en el mes más seco (enero), puede llegar a registrar solo $14 \mathrm{~mm}$. La temperatura oscila entre $18,5^{\circ} \mathrm{C}$ y 5,4 ${ }^{\circ} \mathrm{C}$ que ocurren en los meses de enero y julio respectivamente.

La vegetación existente corresponde al Tipo Forestal RobleHualo, encontrándose renovales de segundo crecimiento con abundante presencia de Nothofagus obliqua y en menor grado Nothofagus alpina (Cruz, Schulze, Honyeman y Cabello, 2006; Donoso, 2015).

Caracterización de los ensayos y la actividad silvicola ejecutada. La actividad silvícola de diciembre del 2011 correspondió a una corta de protección que disminuyó la cobertura arbórea para promover la aparición de regeneración, ajustándose a las prescripciones descritas para este tipo de cortas silvícolas según varios autores (Vita, 1996; Montoya y Mesón, 2004; González, 2005; Serrada, 2008). En el año 2015 se establecieron tres ensayos, diferenciados por su pendiente y cobertura, correspondientes a abierto sin pendiente (AsP), denso sin pendiente (DsP) y denso con pendiente (DcP, pendiente media de 30\%).

Al momento de la evaluación de la regeneración el año 2015, se determinó la posición relativa de cada árbol y cada tocón de los individuos extraídos a partir de coordenadas azimut (ángulo, distancia). A cada árbol le fue medido su diámetro a la altura del pecho (DAP) y cuatro radios de copa. Las mismas variables fueron medidas antes de la corta de protección en el año 2011. Así, fue posible determinar la condición inicial (2011) y la del bosque residual (2015).

Se determinó la densidad de cada ensayo, composición, diámetro cuadrático medio, área basal y cobertura de copa, tanto para antes de la intervención como para la condición residual después de cuatro años.

Caracterización de la regeneración. Cuatro años después de la corta fueron levantadas 69 parcelas para caracterizar la regeneración, de forma circular y radio $3 \mathrm{~m}$, en una red sistemática de 12 metros entre cada una ubicando la primera al azar. En cada parcela se evaluó regeneración por cuadrantes en dirección Norte-Sur y Este-Oeste, para evitar errores de conteo.

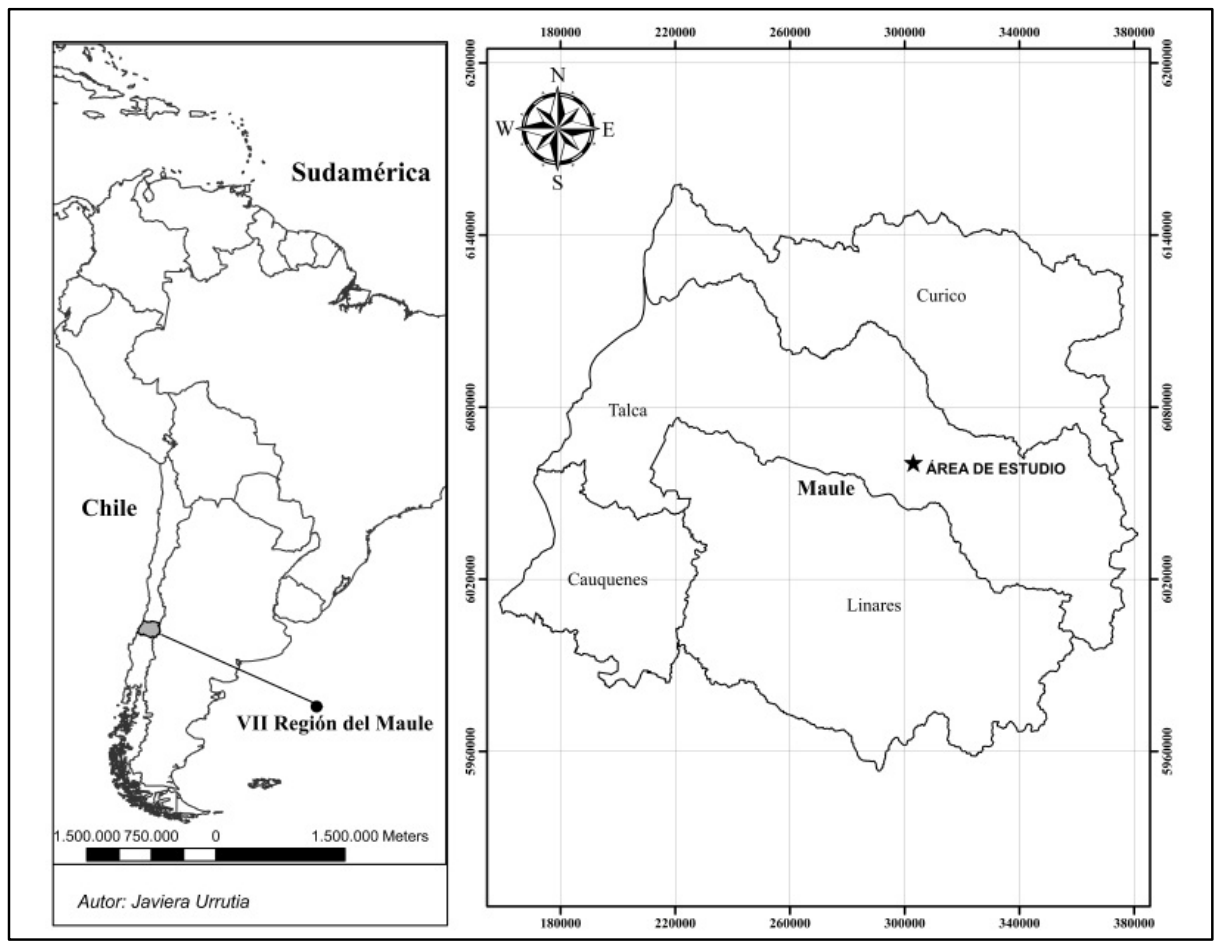

Figura 1. Mapa del área de estudio. 
Las variables recogidas fueron especie, densidad en la parcela y edad de cada individuo. Para escoger como medible una planta, varios autores proponen una altura superior a 0,2 $\mathrm{m}$ (Dezzotti et al., 2003; Rebottaro y Cabrelli, 2011) o 0,5 m (Díaz y Armesto, 2007), o un diámetro de cuello igual o mayor 0,5 cm (Muñoz, Gonzalez, Celedón y Veblen, 2012). Siendo estos criterios insuficientes, para determinar con detalle la temporalidad, se definieron para este caso la siguiente clasificación para cada planta:

$0=$ semillas que estaban recién germinando

$1=$ plantas con más de un año y que no presentaban lignificación en su tallo.

$2=$ plantas con más de dos años y que presentaban tallo lignificado.

$3=$ plantas con más de tres años, que presentaban tallo lignificado y ramificación

El análisis de densidad entre las diferentes situaciones del bosque fue evaluado con prueba de comparaciones múltiples (HSD Tukey), previas pruebas de normalidad y análisis de varianzas (Pardo y Ruíz, 2005).

Para complementar el análisis, se determinó la riqueza florística de las especies arbóreas a través del índice de Simpson (ecuación 1) y la importancia relativa de cada especie con un valor relativo de importancia (VRI) (Del Río, Montes y Cañellas, 2003), basado en densidad y frecuencia (valor máximo 200) (ecuación 2).

$$
\begin{aligned}
& \text { Índice de Simpson }=1-D=1-\sum p_{i}{ }^{2} \\
& \text { Valor de importancia }=\sum(D R+F R)
\end{aligned}
$$

Donde:

$p_{i}$ : $\quad$ proporción de cada especie presente en la muestra

DR : Densidad relativa

FR : Frecuencia relativa

Estas dos últimas variables se calculan como sigue:

$$
\begin{gathered}
D R=\left[\frac{\mathrm{N}^{\circ} \text { individuos dela especie }}{\mathrm{N}^{\circ} \text { de individuos de todas las especies }}\right] \times 100 \\
F R=\left[\frac{\mathrm{N}^{\circ} \text { de ocurrencia de una especie }}{\mathrm{N}^{\circ} \text { de ocurrencia de todas las especies }}\right] \times 100
\end{gathered}
$$

\section{ResultadOS}

\section{Caracterización de los ensayos y la actividad silvícola ejecutada}

El ensayo abierto sin pendiente (AsP) poseía una composición pura (Fig. 2 y 3), que más bien correspondía a una zona sin vegetación arbórea y sin uso productivo, y se mantuvo sin variaciones hasta el 2015. Los ensayos densos (DsP y DcP), al año 2015 tenían una composición mixta, aun cuando N. obliqua poseía una presencia preponderante, tanto en densidad (Tabla 1) como en AB (Tabla 2).

Antes de la corta, los ensayos con bosque tenían a la mayoría de sus individuos en fase de madurez (condición fustal), siendo el DsP algo más denso y con menos desarrollo, respecto a DcP. Siendo bosques dominados por especies intolerantes, podrían definirse en condición de coetáneo fustal.

$\mathrm{El}$ aumento de los DMC residuales respecto a los originales, indican que la extracción afectó principalmente a los individuos menores en los dos ensayos de bosque (DsP y DcP).

La intensidad relativa de la intervención que afectó a los ensayos con bosque (DsP y DcP) fue similar para los atributos de cobertura, AB y densidad (Tabla 3; Fig. 4). No obstante, los valores absolutos residuales son considerablemente diferentes, especialmente en cobertura que quedó con una diferencia de $27 \%$ entre ellos.

\section{Caracterización de la regeneración}

La densidad de regeneración en los ensayos tuvo un comportamiento normal (prueba de K-S y Levene). Las diferencias en las densidades entre los ensayos resultaron estadísticamente significativas (** $\mathrm{P}<0,05)$. Las pruebas de comparación múltiple (Tukey 0,05), indicaron que los tres ensayos son estadísticamente significativas entre sí (Tabla 4 y 5).

La tendencia entre densidad de regeneración y los atributos dasométricos residuales fue directa entre los tres ensayos, es decir, a mayor cobertura-densidad-área basimétrica, se verificó mayor densidad de regeneración en todas las categorías de edades de las plantas, al menos hasta un máximo de cobertura en torno a $90 \%$ (Tabla 6). El coeficiente de variación total de cada ensayo con bosque es relativamente bajo, lo que indicaría que la regeneración en general es relativamente homogénea en su distribución 


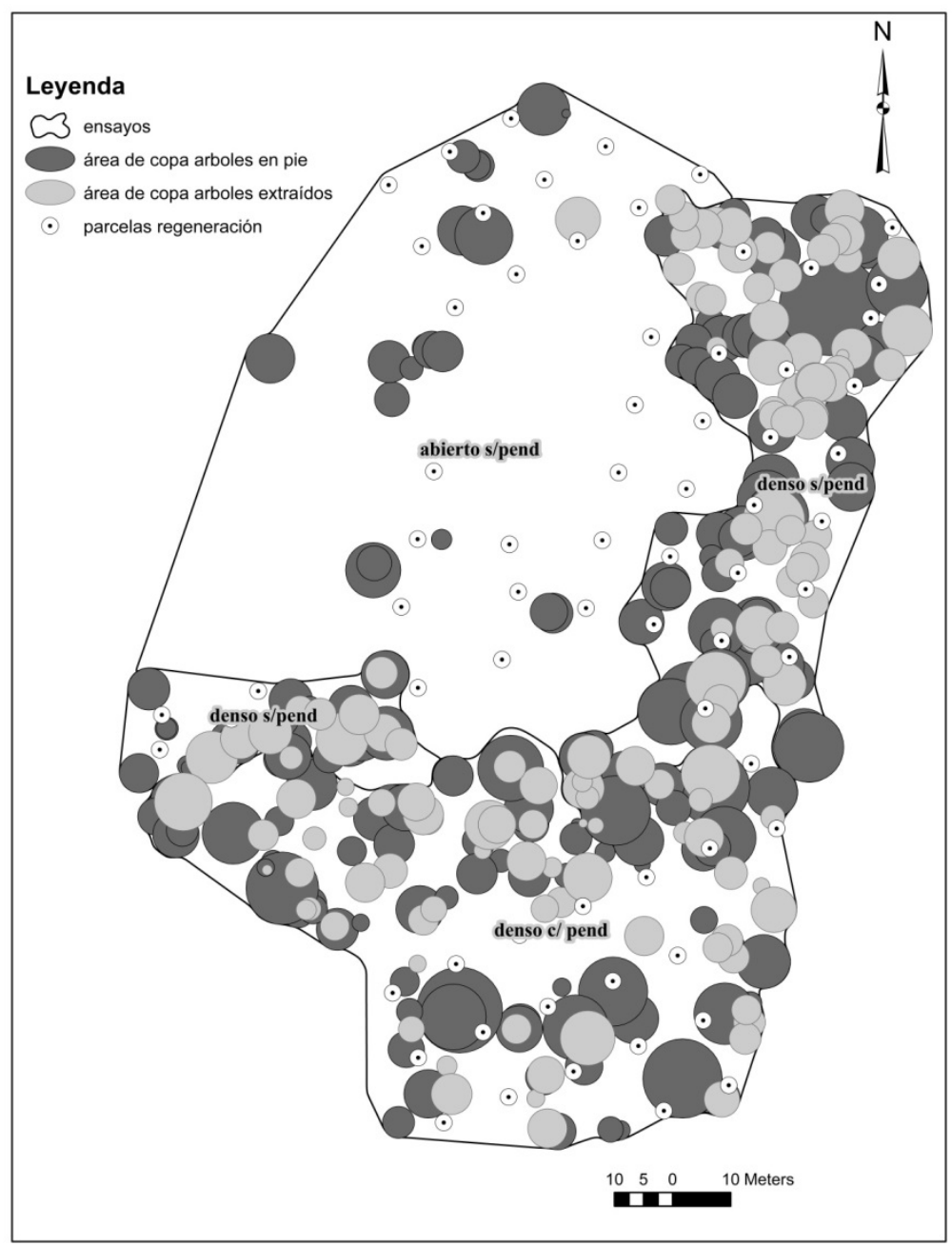

FIGURA 2. Ubicación de las parcelas de regeneración dentro de cada ensayo.
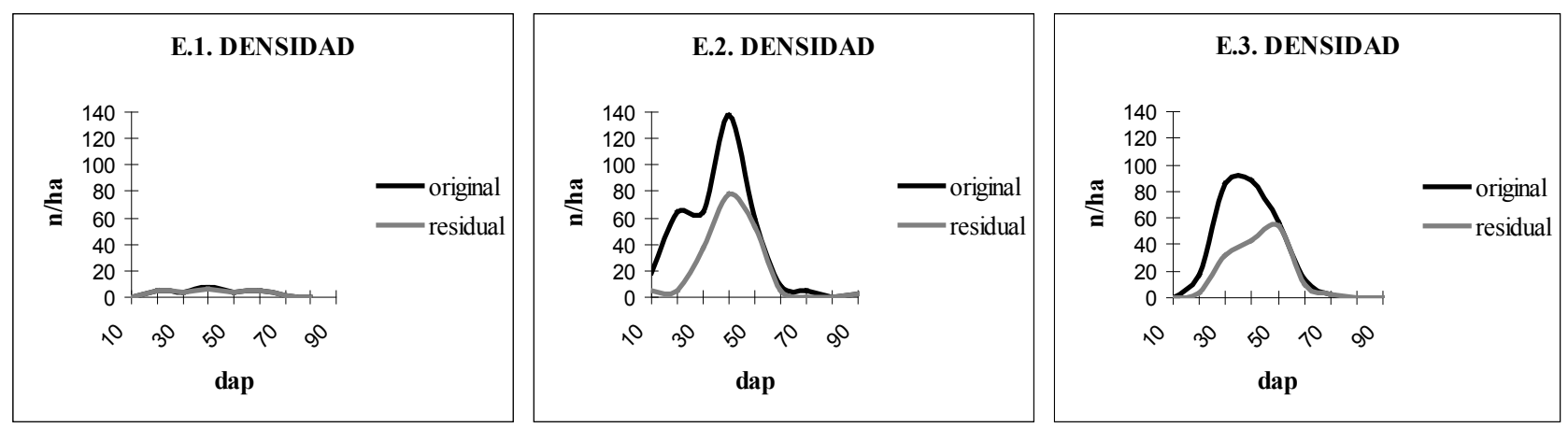

FIGURA 3. Curvas de densidad original y residual para cada ensayo.

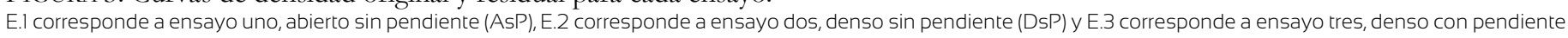
$(\mathrm{DCP})$. 
TABLA 1. Densidad original y residual para cada ensayo, después de cuatro años de la intervención.

\begin{tabular}{|c|c|c|c|c|c|c|c|c|c|c|c|c|c|c|c|}
\hline \multicolumn{16}{|c|}{ Densidad original $\left(n h^{-1}\right)$} \\
\hline & No & Total & No & $\mathrm{Ng}$ & Qs & $\mathrm{Ot}$ & Total & No & $\mathrm{Na}$ & $\mathrm{Ga}$ & PI & Qs & $\mathrm{Ca}$ & $\mathrm{Ot}$ & Total \\
\hline Total & 28 & 28 & 287 & 11 & 5 & 62 & 360 & 191 & 4 & 22 & 2 & 11 & 7 & 24 & 262 \\
\hline $\mathrm{DCM}$ & & 44 & 38 & 25 & 40 & 44 & 38 & 41 & 65 & 35 & 30 & 39 & 30 & 36 & 40 \\
\hline Total & 27 & 27 & 172 & 11 & - & - & 184 & 125 & 4 & 7 & 2 & 6 & - & - & 144 \\
\hline DCM & & 44 & 43 & 25 & - & - & 42 & 44 & 65 & 33 & 30 & 37 & - & - & 44 \\
\hline
\end{tabular}

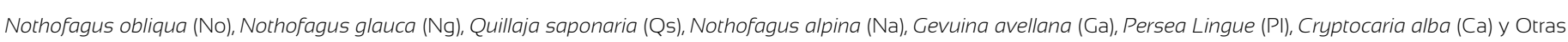
especies (Ot). DMC corresponde al diámetro cuadrático medio.

TABLA 2. Área basal original y residual para cada ensayo.

\begin{tabular}{|c|c|c|c|c|c|c|c|c|c|c|c|c|c|c|}
\hline \multicolumn{15}{|c|}{$A B$ original $\left(m^{2} h a^{-1}\right)$} \\
\hline \multicolumn{2}{|c|}{ Abierto s/pendiente } & \multicolumn{5}{|c|}{ Denso s/pendiente } & \multicolumn{8}{|c|}{ Denso c/pendiente } \\
\hline No & Total & No & $\mathrm{Ng}$ & Qs & Ot & Total & No & $\mathrm{Na}$ & Ga & $\mathrm{PI}$ & Qs & $\mathrm{Ca}$ & Ot & Total \\
\hline Total & 4 & 32 & 1 & 1 & 9 & 42 & 25 & 1 & 2 & 0 & 1 & 1 & 2 & 33 \\
\hline \multicolumn{15}{|c|}{$A B$ residual $\left(m^{2} h a^{-1}\right)$} \\
\hline Total & 25 & 25 & 1 & - & - & 26 & 19 & 1 & 1 & 0 & 1 & - & - & 22 \\
\hline
\end{tabular}

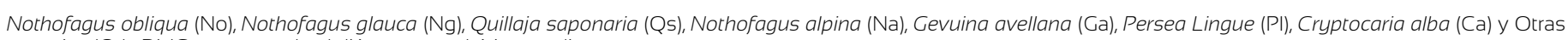
especies (Ot). DMC corresponde al diámetro cuadrático medio.
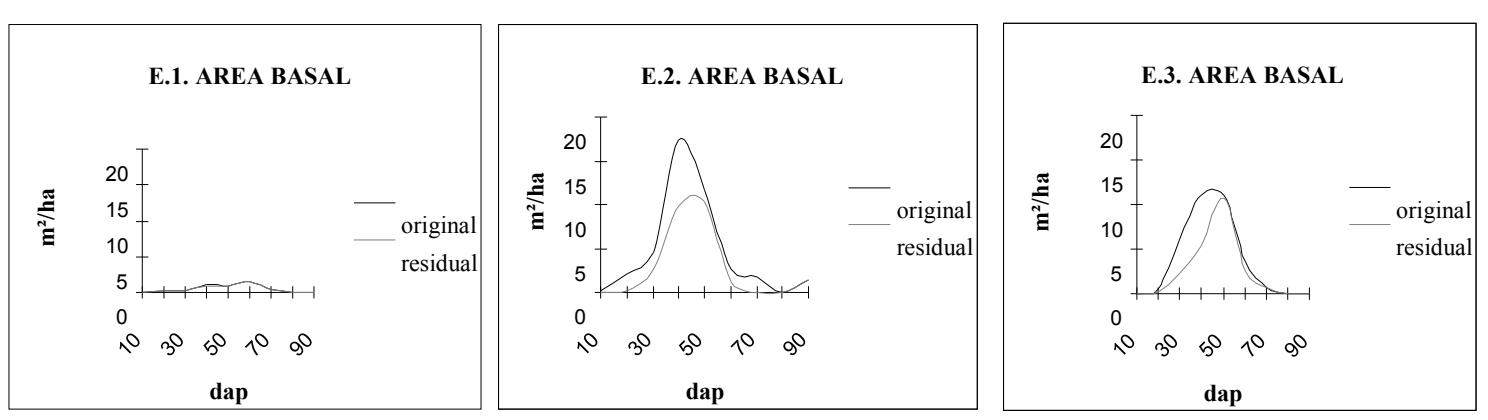

FIGURA 4. Curvas de área basal original y residual para cada ensayo.

E.l corresponde a ensayo uno, abierto sin pendiente (AsP), E.2 corresponde a ensayo dos, denso sin pendiente (DsP) y E.3 corresponde a ensayo tres, denso con pendiente $(\mathrm{DCP})$. 
TABLA 3. Porcentajes de extracción de cobertura, densidad y área basal para cada ensayo.

\begin{tabular}{|c|c|c|c|}
\hline Ensayos & $\begin{array}{c}\text { Abierto } \\
\text { s/pendiente }\end{array}$ & $\begin{array}{c}\text { Denso } \\
\text { s/pendiente }\end{array}$ & $\begin{array}{c}\text { Denso } \\
\text { c/pendiente }\end{array}$ \\
\hline \multicolumn{4}{|c|}{ Cobertura (\%) } \\
\hline Original & 10 & 147 & 89 \\
\hline Residual & 9 & 93 & 60 \\
\hline $\begin{array}{l}\text { Extracción (\% respecto al } \\
\text { Original) }\end{array}$ & $10 \%$ & $37 \%$ & $33 \%$ \\
\hline \multicolumn{4}{|c|}{ Densidad (n ha-1) } \\
\hline Original & 28 & 360 & 262 \\
\hline Residual & 27 & 184 & 144 \\
\hline $\begin{array}{l}\text { Extracción (\% respecto al } \\
\text { Original) }\end{array}$ & $5 \%$ & $49 \%$ & $45 \%$ \\
\hline \multicolumn{4}{|c|}{ Área basal (m² ha-1) } \\
\hline Original & 4,29 & 41,63 & 33,16 \\
\hline Residual & 4,12 & 25,75 & 21,82 \\
\hline $\begin{array}{l}\text { Extracción (\% respecto al } \\
\text { Original) }\end{array}$ & $3,93 \%$ & $38,14 \%$ & $34,19 \%$ \\
\hline
\end{tabular}

TABLA 4. Estadísticas descriptivas para los tres ensayos.

\begin{tabular}{|c|c|c|c|c|c|c|}
\hline \multirow{2}{*}{ Ensayos } & \multirow{2}{*}{$N$} & \multirow{2}{*}{ Media } & \multirow{2}{*}{$\begin{array}{c}\text { Desviación } \\
\text { estándar }\end{array}$} & \multirow{2}{*}{$\begin{array}{c}\text { Error } \\
\text { estándar }\end{array}$} & \multicolumn{2}{|c|}{$\begin{array}{l}95 \% \text { del intervalo de } \\
\text { confianza para la media }\end{array}$} \\
\hline & & & & & Límite inferior & $\begin{array}{l}\text { Límite } \\
\text { superior }\end{array}$ \\
\hline Asp & 25 & 1586 & 2310 & 462 & 633 & 2539 \\
\hline Dcp & 23 & 8332 & 4961 & 1034 & 6187 & 10477 \\
\hline Dsp & 20 & 4592 & 3297 & 737 & 3066 & 6153 \\
\hline Total & 68 & 4757 & 4607 & 559 & 3642 & 5872 \\
\hline
\end{tabular}

TABLA 5. Prueba de comparación múltiple Tukey $\left(\mathrm{p}^{* *}<0,05\right)$.

\begin{tabular}{|c|c|c|c|c|c|}
\hline \multirow[b]{2}{*}{ Prueba } & \multirow[b]{2}{*}{ rodal } & \multirow[b]{2}{*}{$N$} & \multicolumn{3}{|c|}{ Subconjunto para alfa $=0.05$} \\
\hline & & & 1 & 2 & 3 \\
\hline \multirow{4}{*}{ HSD Tukeya,b } & Asp & 25 & 1586 & & \\
\hline & Dcp & 20 & & 4592 & \\
\hline & Dsp & 23 & & & 8332 \\
\hline & Significancia & & 1,000 & 1,000 & 1,000 \\
\hline
\end{tabular}

Se visualizan las medias para los grupos en los subconjuntos homogéneos.

a. Utiliza el tamaño de la muestra de la media armónica = 22,476

b. Los tamaños de grupo no son iguales. Se utiliza la media armónica de los tamaños de grupo. Los niveles de error de tipo I no están garantizados. 
TABLA 6 Cuantificación de la regeneración según densidad, especie y temporada, para cada ensayo.

\begin{tabular}{|c|c|c|c|c|c|c|c|c|c|c|c|c|c|c|c|}
\hline \multicolumn{16}{|c|}{ Densidad de regeneración por ensayo y temporada $\left(n h^{-1}\right)$} \\
\hline \multirow{2}{*}{$\frac{\text { Ensayos }}{\text { Especies }}$} & \multicolumn{5}{|c|}{ AsP (cobertura de copas 10\%) } & \multicolumn{5}{|c|}{$\begin{array}{c}\text { DsP (cobertura de copas residual } \\
90 \%)\end{array}$} & \multicolumn{5}{|c|}{$\begin{array}{c}\text { DcP (cobertura de copas residual de } \\
60 \%)\end{array}$} \\
\hline & E1_O & $\mathrm{E} 1 \_1$ & $\mathrm{E} 1 \_2$ & E1_3 & Total & E2_O & E2_1 & E2_2 & E2_3 & Total & E3_O & E3_1 & E3_2 & E3_3 & Total \\
\hline N. obliqua & 85 & 497 & 283 & 226 & 1091 & 581 & 4361 & 1175 & 355 & 6472 & 141 & 1705 & 566 & 35 & 2448 \\
\hline N. alpina & & & & & & & & & & & 56 & 619 & 231 & 35 & 941 \\
\hline M. boaria & 14 & 156 & 226 & 28 & 424 & 25 & 354 & 530 & 228 & 1137 & & & & & \\
\hline C. alba & & & & & & & 261 & 46 & 62 & 369 & & 248 & 424 & 124 & 796 \\
\hline Otras & & & 28 & 42 & 71 & & 200 & 123 & 31 & 354 & & 248 & 124 & 35 & 407 \\
\hline$P$. lingue & & & & 14 & 14 & & 46 & 46 & 15 & 108 & & 88 & 71 & 0 & 159 \\
\hline P. boldus & & & & & & & 138 & 62 & 15 & 215 & & 141 & 18 & 0 & 159 \\
\hline C. mucronata & & & & & & & 15 & 15 & 0 & 31 & & - & - & - & 0 \\
\hline A. punctatum & & & & & & & & & & & & 18 & 35 & 35 & 88 \\
\hline L. hirsuta & & & 14 & 28 & 42 & & & & & & & & & & \\
\hline Q. saponaria & & & 14 & & 14 & & & & & & & & & & \\
\hline Total & 99 & 652 & 538 & 297 & & 606 & 5176 & 1874 & 676 & & 197 & 2820 & 1345 & 230 & \\
\hline Total Ensayo & & & 1586 & & & & & 8332 & & & & & 459 & & \\
\hline
\end{tabular}

AsP: abierto sin pendiente; Dsp: Denso sin pendiente; DcP: Denso con pendiente; El_O corresponde a Ensayo uno (1), temporada de plantas "semillas recién germinando" (O): El_l: Ensayo 1 plantas de la temporada 1 (plantas con más de un año y que no presentaban lignificación en su tallo); El_2: Ensayo 1 plantas de la temporada 2 (plantas con más de dos años y que presentaban tallo lignificado); El_3: Ensayo l con plantas de la temporada 3 (plantas con más de tres años, que presentaban tallo lignificado y ramificación); . Otras corresponden a la suma de las especies desglosadas al margen derecho. Otras: Persea lingue Ruiz et. Pav.; Peumus boldus Mol.; Citronella mucronata (Ruiz et. Pav.); Aextoxicon punctatum (Ruiz et. Pav.); Lomatia hirsuta Diels ex J.F.Macbr.; Quillaja saponaria Mol.

espacial. El ensayo abierto en cambio, con un alto coeficiente de variación total y por especie, indicando que hasta los cuatro años el reclutamiento es heterogéneo.

$N$. alpina únicamente presentó regeneración en el ensayo donde hubo adultos de su especie (DcP). Esta particularidad podría deberse al mayor contenido de humedad respecto al bosque plano (DsP), siendo evidencia de ello la presencia de Aextoxicon punctatum R. et P. en este ensayo, ya que $A$. punctatum ha sido descrita como especie fuertemente tolerante y con alta afinidad a zonas húmedas (Donoso, 2006). Por otra parte, y suponiendo la existencia de un gradiente de contenido de humedad entre los tres ensayos, se podría afirmar que Quillaja saponaria Mol., Lomatia birsuta (Lam.) Diels ex Macbr. y Maytenus boaria Mol. son las especies favorecidas por la sequedad en el área, teniendo $M$. boaria más plasticidad respecto a la luminosidad al establecerse tanto en áreas abiertas como bajo dosel. Se debe destacar, por otra parte, la participación de Cryptocarya alba Mol. Looser en aquellos ensayos con cobertura. N. obliqua definitivamente es la especie dominante en todos los casos, presentando más del doble de la densidad en el ensayo sin pendiente (DsP) respecto al ubicado en pendiente (DcP).

El comportamiento de regeneración varía según la temporada y las especies. N. obliqua y N. alpina y Peumus boldus Mol., por ejemplo, tuvieron el máximo de densidad en plantas de la categoría "más de un año" (Fig. 5; E1_1; E2_1; E3_1), N. obliqua, en particular, mostró este comportamiento en los tres ensayos; mientras que $M$. boaria, en general, y $C$. alba, en el bosque denso con pendiente, presentaron sus máximos en la categoría de "plantas de más de dos años", en los ensayos donde regeneraron (Tabla 6). Persea lingue, por otra parte, presenta una densidad constante para plantas de una temporada y otra (Tabla 6).

El comportamiento descrito, podría interpretarse como un reclutamiento precoz de $M$. boaria, C. alba y A. punctatum, respecto al de N. obliqua, N. alpina y C. alba, mientras que P. lingue tendría un reclutamiento más bien constante en el tiempo y hasta el momento de la evaluación. 


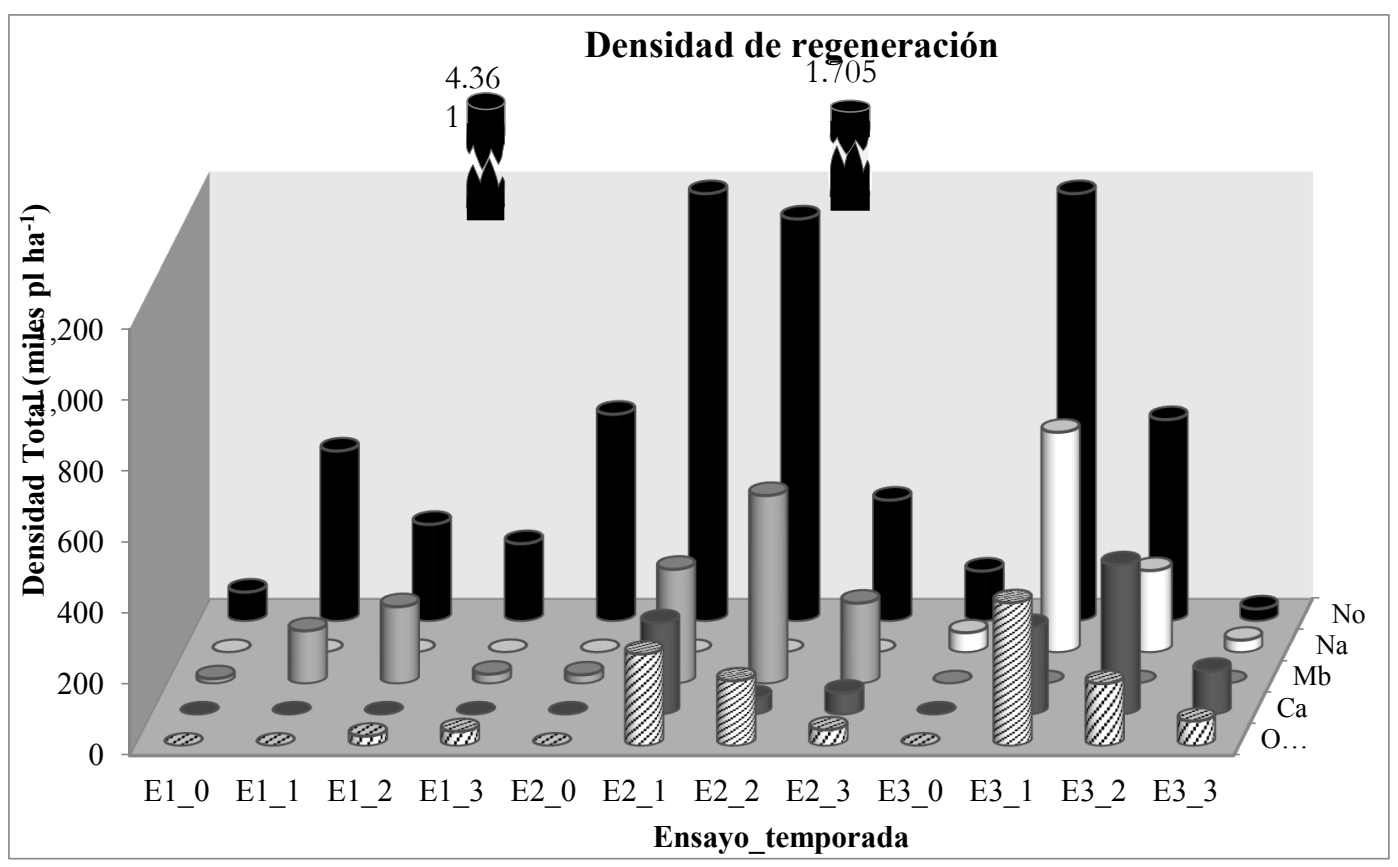

FIGURA 5. Representación de la regeneración según densidad, especie y temporada en la que se encuentran, para cada ensayo.

El_O: ensayo I(AsP) con su respectiva temporada O, 1, 2 y 3 y así sucesivamente. No; Nothofagus obliqua; Na: Nothofagus alpina; Mb: Maytenus boaria; Ca: Cryptocaria alba; Otras: Persea lingue; Peumus boldus; Citronella mucronata; Aextoxicon punctatum; Lomatia hirsuta; Quillaja saponaria.

En todos los ensayos es notoria la importancia relativa de $N$. obliqua (VRI, Tabla 7), que tiene altos valores de densidad relativa como de frecuencia relativa, lo que representaría la mejor distribución en el espacio y mayor presencia en los tres ensayos, siendo destacable que en el bosque denso sin pendiente (DsP) ocupa tres cuartas partes de la regeneración relativa total, a pesar que la cobertura residual alcanzó 90\%. Solamente C. alba alcanza una presencia y distribución notoria en el ensayo con pendiente (DcP), el que además tiene una cobertura de 60\%. M. boaria sería la tercera especie con valores destacables de importancia en los tres ensayos. El resto de las especies tienen más bien una importancia discreta.

La diversidad estimada resultó similar en las áreas planas, no obstante sus diferencias en densidad total. El ensayo de bosque con pendiente (DcP) ofrece mayor diversidad que los anteriores, debido a que en los casos de los ensayos planos únicamente dos especies arrojan importancias relativas fuertes ( $N$. obliqua y $M$. boaria), mientras que en el ensayo con pendiente, son tres las especies con alta importancia (N. obliqua, N. alpina y C. alba). Así, las diferencias descritas en el índice de Simpson, no se deben a la cantidad de especies, que en este caso son similares en número entre los tres ensayos, sino a las diferencias de peso en la importancia que cada una tiene, es decir, la diversidad entre casos, para el mismo número de especies, será mayor si la importancia entre ellas es similar.

El conjunto de atributos de valor relativo de importancia (Tabla 7), diversidad, densidad total, permite indicar que a cuatro años de la intervención, las coberturas residuales mayores a $60 \%$, pueden favorecer una regeneración mixta con densidades mayores a ocho mil plantas por hectárea, respecto a áreas descubiertas que ofrecieron cerca de 1500 plantas por hectárea, con una composición igualmente mixta pero con especies diferentes, siendo siempre N. obliqua una especie dominante en la nueva generación. En el mismo sentido, y considerando la importancia relativa de las especies regeneradas y la diversidad en cada caso, una cobertura cercana a $60 \%$ en zonas con pendiente, otorgan una composición mixta robusta, es decir, varias especies y cada una con mejor importancia en la composición. 
TABLA 7. Valor de importancia relativa VRI (máx. 200) y diversidad de Simpson estimado para la regeneración en cada ensayo.

\begin{tabular}{|c|c|c|c|c|c|c|c|c|c|}
\hline \multirow[b]{2}{*}{ especie } & \multicolumn{3}{|c|}{$\begin{array}{c}\text { AsP } \\
\text { (cobertura de C. 10\%) } \\
\end{array}$} & \multicolumn{3}{|c|}{$\begin{array}{c}\text { DsP } \\
\text { (cobertura de C. residual 90\%) }\end{array}$} & \multicolumn{3}{|c|}{$\begin{array}{c}\text { DcP } \\
\text { (cobertura de C. residual 60\%) }\end{array}$} \\
\hline & $\begin{array}{c}\text { densidad } \\
\text { relativa }\end{array}$ & $\begin{array}{l}\text { frecuenci } \\
\text { a relativa } \\
\end{array}$ & VRI & $\begin{array}{c}\text { densidad } \\
\text { relativa }\end{array}$ & $\begin{array}{c}\text { frecuencia } \\
\text { relativa }\end{array}$ & VRI & $\begin{array}{c}\text { densidad } \\
\text { relativa }\end{array}$ & $\begin{array}{c}\text { frecuencia } \\
\text { relativa }\end{array}$ & VRI \\
\hline N. obliqua & 62,2 & 42,3 & 104,5 & 74,7 & 41,8 & 116,5 & 45,7 & 28,3 & 74,0 \\
\hline N. alpina & - & - & - & - & & - & 17,8 & 15,1 & 32,9 \\
\hline M. boaria & 22,1 & 19,2 & 41,3 & 14,4 & 16,4 & 30,7 & - & & - \\
\hline C. $a l b a$ & - & - & - & 4,3 & 7,3 & 11,5 & 25,3 & 28,3 & 53,6 \\
\hline P. lingue & 2,8 & 5,8 & 8,5 & 2,1 & 10,9 & 13,0 & 5,0 & 9,4 & 14,4 \\
\hline P. boldus & 1,8 & 7,7 & 9,5 & 3,2 & 12,7 & 16,0 & 3,1 & 13,2 & 16,3 \\
\hline C. mucronata & - & - & - & 0,3 & 3,6 & 4,0 & - & & - \\
\hline A. punctatum & - & - & - & - & - & - & 2,8 & 3,8 & 6,6 \\
\hline L. hirsuta & 8,3 & 15,4 & 23,7 & - & - & - & - & & - \\
\hline Q. saponaria & 2,3 & 7,7 & 10,0 & 0,7 & 3,6 & 4,3 & 0,3 & 1,9 & 2,2 \\
\hline $\begin{array}{l}\text { Lithraea caustica } \\
\text { Molina. }\end{array}$ & 0,5 & 1,9 & 2,4 & 0,3 & 3,6 & 4,0 & - & & - \\
\hline Total ensayo & & & 200,0 & & & 200,0 & & & 200,0 \\
\hline Ind Simpson & & & 0,56 & & & 0,42 & & & 0,69 \\
\hline Densidad ensayos & & & 1586 & & & 8332 & & & 4592 \\
\hline
\end{tabular}

\section{DISCUSIÓN}

La presencia de $N$. obliqua en regeneración en todas las intensidades de cobertura en los ensayos, pareciera indicar que esta especie posee una amplia plasticidad respecto a la luminosidad al establecerse. Resultados similares fueron publicados por Martínez-Pastur et al. (1999), relativos a la presencia de $N$. obliqua latente en bosques adultos cubiertos. No obstante, las densidades generales y en especial la de N. obliqua fueron significativamente menores a la hallada por otros autores que analizaron áreas descubiertas por más tiempo. En efecto, Dezzotti et al. (2003) indican densidades de hasta 90 mil plantas por hectárea, a los ocho años. Sola et al. (2015) en la reserva Nacional Lanín, registró más de 12 mil plantas por hectárea después de cinco años de la apertura del dosel. El reclutamiento en los tres ensayos pareciera estar activo al encontrarse plántulas en los primeros estados del establecimiento.

Es posible que la reducida densidad en cuatro años se deba a la influencia combinada del período de sequía que ha afectado a la zona desde antes de haberse establecido los ensayos $\left(\mathrm{CR}^{2}\right.$ (Centro de Ciencia del Clima y la Resilencia), 2015) y la propia variación que Nothofagus posee en su dinámica regenerativa. Al respecto, Rosenfeld (1972) describe variaciones en la regeneración de Nothofagus pumilio (Poepp. et Endl.) (Lenga) desde 40 mil a 470 mil plantas por hectárea después de tres años a cinco años de las intervenciones. Un efecto destacable es el importante grado de participación de un conjunto de especies de diversa tolerancia e incluso de ecosistemas forestales cuya preponderancia ocurre más al norte, como es el caso de $Q$. saponaria y C. alba. En efecto, la importancia relativa del grupo de 
especies diferentes a $N$. obliqua se concentró en torno a 50\% del total. Si bien otros autores han encontrado que aperturas del dosel permiten que $N$. obliqua regenere en compañía de otras especies tolerantes, como L. philippiana (Pollmann, 2004) y P. lingue (Weinberger y Ramírez, 1999), no fue posible encontrar estudios que mencionen la importancia de especies arbóreas mediterráneas regenerando en bosques templados. Así, esta importante participación podría ser momentánea debido a la sequedad que implica una apertura del dosel, y que luego dichas especies mediterráneas podrían desaparecer, o bien podría ser un fenómeno reciente derivado de un cambio global en el clima de la zona, en cuyo caso las composiciones futuras podrían cambiar con mayor participación de las especies arbóreas mediterráneas. Si este fuera el caso, la dinámica de la migración del bosque mediterráneo hacia el sur, se estaría produciendo en intermitencias temporales coincidentes con eventos de sequía regional. Si bien no fueron encontrados antecedentes que expliquen esta dinámica, los antecedentes existentes de la autoecología de las especies de climas más secos (Cabello y Donoso, 2013; Donoso, Cabello y Escobar, 2013; Cruz et al., 2006), coinciden en describir su distribución en los bosques más secos de la zona norte del país, siendo los bosques $N$. obliqua su distribución límite sur, que coincide con el aumento de las precipitaciones en Chile.

Las diferencias estadísticas encontradas entre el ensayo abierto y los densos contradicen las afirmaciones de varios autores que indican que la respuesta de estos bosques a la fuerte apertura del dosel provocada por destrucción es la aparición de rodales puros de N. obliqua (Veblen y Ashton, 1978; Veblen et al., 1980; Donoso, 2015). No obstante, estos autores no indican los tiempos requeridos para dicho establecimiento y podría ocurrir que el tiempo que duró este estudio, no haya sido suficiente.

Respecto de los ensayos en bosques densos en que también existen diferencias estadísticamente significativas, las densidades notoriamente distintas fueron aquellas de N. obliqua, con más del doble de plantas en la zona sin pendiente y mayor cobertura residual, lo que además es consistente con su mayor importancia relativa en dicho ensayo, respecto del ensayo con pendiente (Tablas 6 y 7). Los estudios previos revisados no permiten explicar claramente estas diferencias. Por una parte, diversos autores describen la presencia de altas densidades de regeneración de N. obliqua bajo dosel, como es el caso de ambos ensayos, pero no detallan específicamente diferencias ocurridas ni por cobertura ni por pendiente (Martínez-Pastur et al., 1999; Dezzotti et al., 2003). Un hecho probable, no obstante, es la influencia marcada de la mayor humedad que pudiera tener el ensayo con pendiente, evidenciado por la presencia de $A$. punctatum, cuya autoecología indica que solamente se establece en sitos con alta humedad (Donoso, 2015).

Los máximos en la instalación de las especies mediterráneas (M. boaria y C. alba), ocurrieron en años cercanos a la corta, antes que $N$. obliqua, y que podría ser consecuencia de la coincidencia de la corta con el inicio del período de sequía. Donoso, et al. (2013) en su revisión de la autoecología de la especie indican que $M$ boaria, sería escaso o ausente en bosques de $N$. obliqua, siendo más frecuente en bosques xéricos de más al norte. No fueron encontrados antecedentes que describan la oportunidad en la que aparece la especie luego de alguna corta o apertura del dosel. En todo caso, en vistas de fortalecer acciones silvícolas sobre la masa futura, se podría afirmar que en la dinámica del establecimiento hasta los cuatro años, existen especies que se instalan hasta sus máximos tempranamente, otras requieren plazos mayores a dos años (N. obliqua), y un tercer grupo que presenta un flujo similar hasta los cuatro años después de la corta La recopilación de antecedentes relativos a la regeneración de $N$. obliqua, nada indican de esta dinámica (Martínez-Pastur et al., 1999; Dezzotti et al., 2003; Muñoz et al., 2012; González et al., 2015).

Por otra parte, el hecho que la intensidad de corta fuera similar en valores relativos en los dos ensayos con bosque, y que determinara bosques residuales claramente diferentes en sus atributos en valores absolutos, permite recomendar al menos una revisión de las indicaciones que fijen las extracciones en porcentajes de las existencias iniciales, y que pueden determinar diferencias sustantivas en el establecimiento de la regeneración (Conaf, 1980; Conaf / Gesellschaft für. Technische Zusammenarbeit [GTZ], 1998; Instituto Forestal [Infor]Fundación Chile, 2008). Sería recomendable más bien fijar condiciones en las que debería quedar el bosque residual en valores absolutos, independiente de la condición inicial.

La alta diversidad encontrada al aplicar el método silvícola de cortas de protección permitiría un mejor control de la composición de la regeneración futura, o sería una opción de 
formar bosques mixtos, en contraposición a los renovales puros que varios autores indican que se formarían en áreas completamente descubiertas en la zona de Nothofagus (Veblen y Ashton, 1978; Veblen, 1985; Donoso y Lara, 1999).

Finalmente es posible afirmar que la hipótesis planteada, respecto de la utilidad de la corta de protección para favorecer la regeneración en bosques de Nothofagus obliqua, no solo se cumple, sino que además otorga la ventaja de propiciar una regeneración mixta. No obstante, es posible que cuatro años sea un tiempo insuficiente para considerar como regenerado el bosque, en consideración que otros autores proponen como establecida una planta cuando su altura supera 0,2 m (Dezzotti et al., 2003; Rebottaro y Cabrelli, 2011) o 0,5 m (Díaz y Armesto, 2007) o su diámetro de cuello es igual o mayor 0,5 cm (Muñoz et al., 2012), condiciones que aún no se alcanzaban en el momento de este estudio.

\section{CONCLUSIONES}

Coberturas residuales mayores a $60 \%$ permitieron un establecimiento de más de 8 mil plantas por hectárea después de cuatro años, que puede ser considerado bajo respecto de otros estudios. Una causa probable es el intenso período de sequía que coincidió con el estudio. Estas condiciones de sequía podrían justificar también, que cerca de la mitad de la densidad de regeneración fuera de especies de bosques mediterráneos, que en Chile se ubican en zonas más secas, al norte y más bajo en altitud, respecto de la distribución de Nothofagus. En este sentido, sería interesante estudiar si los ciclos de sequía podrían constituir flujos de especies mediterráneas migrando hacia la zona actual de los bosques templados.

La gran diversidad de especies en la regeneración que provoca una corta semillera, puede ser una ventaja silvícola respecto de tala rasa, que, imitando desastres naturales, generan renovales puros. En consecuencia, se podría afirmar que la hipótesis respecto de la utilidad de esta corta para establecer la regeneración en bosques de $N$. obliqua no solo se cumple, sino que otorga la opción de una regeneración diversificada. No obstante, a cuatro años de efectuada la corta no existe suficiente densidad como para proponer una nueva corta, así como tampoco se podrían considerar que las plantas de la regeneración están completamente establecidas.

\section{RECONOCIMIENTOS}

Se agradece la colaboración del equipo de la Corporación Nacional Forestal de la región del Maule.

\section{REFERENCIAS}

Cabello, A., \& Donoso, C. (2013). Cryptocarya alba. En C. Donoso, \& C. Donoso (Ed.), Autoecología de las especies arbóreas de los bosques templados de Chile y Argentina (pág. 688). Valdivia: Marisa Cuneo.

Centro de Ciencia del Clima y la Resilencia. (2015). La megasequía 20102015. Centro de Ciencia del Clima y la Resilencia. Recuperado el 26 de nov de 2015

Corporación Nacional Forestal, CL [Conaf] (1980). Reglamento Técnico del D. L. $N^{o}$ 701. Santiago: Conaf.

Corporación Nacional Forestal, CL [Conaf] / Gesellschaft für. Technische Zusammenarbeit [GTZ] (1998). Experiencia silvicultural del bosque nativo chileno. (P. del Fierro, Ed.) Santiago: Publicaciones Lo castillo.

$\mathrm{CR}^{2}$ (Centro de Ciencia del Clima y la Resilencia). (6 de abril de 2015). Informe a la Nación La mega sequía 2010-2015 una lección para el futuro. Obtenido de //www.cr2.cl/megasequia/

Cruz, P., Schulze, C., Honyeman, P., \& Cabello, A. (2006). Quillaja saponaria. En C. Donoso, Las especies arbóreas de los bosques templados de Chile y Argentia (págs. 545-555). Valdivia: Marisa Cuneo.

Del Río, M., Montes, F., \& Cañellas, I. M. (2003). Índices de diversidad estructural en masas forestales. Invest. Agrar.: Sist. Recur. For., 12(1), 159-176.

Dezzotti, A., Sbrancia, R., Rodríguez-Arias, M., Roat, D., \& Parisi, A. (2003). Regeneración de un bosque mixto de Nothofagus (Nothofagaceae) después de una corta selectiva. Revista Chilena de Historia Natural, 76(4), 591-602. doi: 10.4067/S0716078X2003000400004

Díaz, M., \& Armesto, J. (2007). Limitantes físicos y bióticos de la regeneración arbórea en matorrales sucesionales de la Isla Grande de Chiloé, Chile. Rev. chil. hist. nat, 13-26.

Donoso, C. (2006). Las especies arbóreas de los bosques templados de Chile y Argentina. Autoecología. Valdivia: Marisa Cuneo.

Donoso, C. (2015). Estructura y dinámica de los bosques del cono sur de américa. Santiago: Ediciones U Mayor.

Donoso, C., \& Lara, A. (1999). Silvicultura de los bosques nativos de Chile. Santiago: Universitaria.

Donoso, C., Cabello, A., \& Escobar, B. (2013). Maytenus boaria. En C. Donoso, \& C. Donoso (Ed.), Las especies arbóreas de los bosques templados de Chile y Argentina. Autoecología (pág. 688). Valdivia: Marisa Cuneo.

González, J. (2005). Introducción a la Selvicultura General. Ponferrada: Universidad de León.

González, M., Donoso, P., \& Zejner, P. (2015). Tree fall gaps and patterns of tree recruitment and growth in Andes old-growth in south-central Chile. Bosque, 36(3), 383-393. doi:10.4067/S071792002015000300006

Grosse, H., \& Quiroz, I. (1998). Silvicultura de los bosques de segundo crecimiento de roble, raulí y coigüe en la región centro-sur de 
Chile. En C. Donoso, \& A. Lara, Silvicultura de los bosques nativos de Chile (pág. 421). Santiago, Chile: Editorial Universitaria.

INFOR-Fundación Chile. (2008). Guías Expertas de Mejores Prácticas de Manejo Sustentable para Renovales de Nothofagus.

Martínez-Pastur, G., Peri, P., Fernández, M., \& Staffieri, G. (1999). Desarrollo de la regeneración a lo largo del ciclo del manejo forestal de un bosque de Nothofagus pumilio. Bosque, 20(2), 3946.

Martínez-Velásquez, A. (1998). Silvicultura práctica en renovales puros y mixtos, y bosques remanentes originales del tipo forestal robleraulí-coigüe. En C. Donoso, \& A. Lara, Silvicultura de los bosques nativos de Chile (págs. 145-175). Santiaqgo, Chile: Editorial Universitaria.

Merkel, Alexander. (18 de febrero de 2018). climate-data. Obtenido de https:/ / es.climate-data.org

Montoya, J., \& Mesón, G. (2004). Selvicultura (1141 ed., Vol. II). Madrid: Mundiprensa.

Muñoz, A., Gonzalez, M., Celedón, C., \& Veblen, T. (2012). Respuesta inicial de la regeneración arbórea luego de la floración y muerte de Chusquea culeou (Poaceae) en bosques andinos del centro-sur de Chile. Bosque, 153-162. doi:10.4067/S071792002012000200005

Otero, L., \& Monfil, T. (1999). Silvicultura de los bosques del tipo forestal Coihue Raulí Tepa. En C. Donoso, \& A. Lara, Silvicultura de los bosques nativos de Chile (págs. 245-269). Santiago, Chile: Editorial Universitaria.

Pardo, A., \& Ruiz, A. (2005). Guía para el análisis de datos. Madrid: Universidad Autónoma de Madrid.

Philip, G., \& Watson, D. (1982). A Precise Method for Determining Contoured Surfaces. Australian Petroleum Exploration Association Journal, 22, 205-212.

Pollmann, W. (2004). Regeneration dynamics and life history differences in southern Chilean Nothofagus forests: a synthesis. Plant Ecology, 174, 353-369. doi:10.1023/B:VEGE.0000049114.58663.6d

Rebottaro, S., \& Cabrelli, D. (2011). Regeneración natural de Pinus elliottii en claros silvícolas: dinámica poblacional durante siete años. Madera y Bosques, 49-70. doi: 10.21829/myb.2011.1711154.

Rosenfeld, J. (1972). Desarrollo de la regeneración de Rauli y Coibue bajo diferentes grados de luminosidad. Universidad Austral. Valdivia: UACh.
Serrada, R. (2008). Apuntes de selvicultura. Madrid: Universidad Politécnica de Madrid.

Sola, G., Attis, H., Chauchard, L., \& Gallo, L. (2015). Efecto del manejo silvicultural sobre la regeneración de un bosque de Nothofagus dombeyi, N. alpina y N. obliqua en la Reserva Nacional Lanín (Argentina). Bosque, 36(1), 113-120. doi:10.4067/S071792002015000100012

Veblen, T. (1985). Forest development in tree-fall gaps in the temperate rain forests in Chile. Nat. Geogr. Res, 1, 161-184.

Veblen, T., \& Ashton, D. (1978). Catastrophic influences on the vegetation of the Valdivian Andes, Chile. Vegetation, 36(3), 149167.

Veblen, T., Schlegel, F., \& Escobar, B. (1980). Structure and dynamics of old-growth Nothofagus forest in the Valdivian Andes, Chile. Journal of Ecology, 68, 1-31.

Vita, A. (1996). Los tratamientos silviculturales. Santiago: Universidad de Chile.

Watson, D., \& Philip, G. (1985). A Refinement of Inverse Distance Weighted Interpolation. Geoprocessing, 2, 315-327.

Weinberger, P., \& Ramírez, C. (1999). Sinecología de la regeneración natural del Raulí (Nothofagus alpina) Fagaceae, Magnoliopsida. Revista Chilena de Historia Natural, 72, 337-351.

Manuscrito recibido el: 16 de mayo de 2017

Aceptado el: 21 de febrero de 2018

Publicado el: 22 de agosto de 2018

Este documento se debe citar como:

Curz, P., \& Urrutia, J. (2018). Efecto de una corta de protección sobre el establecimiento de regeneración en bosque de Nothofagus. Madera y Bosques, 24(2), e2421558. doi: 10.21829/myb.2018.2421558

Madera y Bosques por Instituto de Ecología, A.C. se distribuye bajo una Licencia Creative Commons Atribución-NoComercialCompartirlgual 4.0 Internacional. 